\title{
Identification of quantitative trait loci underlying milk traits in Spanish dairy sheep using linkage plus combined linkage disequilibrium and linkage analysis approaches
}

\author{
E. Garcia-Gámez, ${ }^{1}$ B. Gutiérrez-Gil, ${ }^{1}$ A. Suarez-Vega, L. F. de la Fuente, and J. J. Arranz ${ }^{2}$ \\ Departamento de Producción Animal, Facultad de Veterinaria, Universidad de León, 24071 León, Spain
}

\begin{abstract}
In this study, 2 procedures were used to analyze a data set from a whole-genome scan, one based on linkage analysis information and the other combing linkage disequilibrium and linkage analysis (LDLA), to determine the quantitative trait loci (QTL) influencing milk production traits in sheep. A total of 1,696 animals from 16 half-sib families were genotyped using the OvineSNP50 BeadChip (Illumina Inc., San Diego, CA) and analysis was performed using a daughter design. Moreover, the same data set has been previously investigated through a genome-wide association (GWA) analysis and a comparison of results from the 3 methods has been possible. The linkage analysis and LDLA methodologies yielded different results, although some significantly associated regions were common to both procedures. The linkage analysis detected 3 overlapping genome-wise significant QTL on sheep chromosome (OAR) 2 influencing milk yield, protein yield, and fat yield, whereas 34 genome-wise significant QTL regions were detected using the LDLA approach. The most significant QTL for protein and fat percentages was detected on OAR3, which was reported in a previous GWA analysis. Both the linkage analysis and LDLA identified many other chromosome-wise significant associations across different sheep autosomes. Additional analyses were performed on OAR2 and OAR3 to determine the possible causality of the most significant polymorphisms identified for these genetic effects by the previously reported GWA analysis. For OAR3, the analyses demonstrated additional genetic proof of the causality previously suggested by our group for a single nucleotide polymorphism located in the $\alpha$-lactalbumin gene $(L A L B A)$. In summary, although the results shown here suggest that in commercial dairy populations, the LDLA method exhibits a higher efficiency to map QTL
\end{abstract}

\footnotetext{
Received March 18, 2013.

Accepted May 22, 2013.

${ }^{1}$ Both authors contributed equally to this work.

${ }^{2}$ Corresponding author: jjarrs@unileon.es
}

than the simple linkage analysis or linkage disequilibrium methods, we believe that comparing the 3 analysis methods is the best approach to obtain a global picture of all identifiable QTL segregating in the population at both family-based and population-based levels.

Key words: sheep, milk trait, quantitative trait loci, combined linkage disequilibrium and linkage analysis method

\section{INTRODUCTION}

For several years, mapping the QTL of economic interest in livestock species using genetic markers was based on pedigree or family information (Georges, 2007). In commercial dairy populations, paternal halfsib pedigrees, generated using AI, have been used to investigate the co-segregation of genetic markers and QTL in large half-sib families (daughter or granddaughter designs; Weller et al., 1990; Georges et al., 1995). More recently, due to the development of nextgeneration sequencing technologies, the genomes of many livestock species have been sequenced. These advances have been accompanied by the availability of cost-efficient, high-throughput genotyping platforms that allow the investigation of the genomes at a high resolution to identify genetic variants for traits of economic and biological interest.

In dairy cattle, since the availability of SNP array panels, genome-wide association (GWA) analyses have replaced traditional linkage mapping studies (Mai et al., 2010; Schopen et al., 2011). These GWA studies, which are ideally conducted in unrelated individuals, and the increased marker density offered by the highthroughput genotyping platforms allow the analysis of population-wide linkage disequilibrium (LD). Because LD generally extends over much shorter distances than the genetic linkage analyzed in classical QTL detection studies based on linkage analysis, these LD-based analyses allow the identification of reduced confidence intervals for the QTL location compared with estimates based on classical linkage analyses (Weller and Ron, 2011). 
Nevertheless, the power of GWA studies depends on the extent of the LD across the genome (Andersson, 2009). Investigations in cattle have demonstrated that the $\mathrm{LD}$ varies along the genome and that a certain degree of LD exists between markers on different chromosomes (Farnir et al., 2002; McRae et al., 2002). For the populations structured as half-sib families, several studies have suggested that the combination of LD and linkage analysis information (LDLA) enhances the feasibility of fine-mapping QTL regions (Meuwissen et al., 2001, 2002; Farnir et al., 2002). Instead of using single SNP information, the LDLA approach is based on marker haplotypes and the construction of an identity-by-descent matrix. In a half-sib design, the linkage information is provided by the paternal haplotypes of the progeny, whereas both the paternal and maternal haplotypes of the progeny provide the LD information. To implement LDLA successfully in this type of experimental design, the maternal haplotypes should be a good representation of the whole-population haplotypes (Meuwissen et al., 2002). Additionally, an optimal number of half-sib families should be included in the experiment. In a simulation study, Roldan et al. (2012) demonstrated that the analysis of 15 to 50 mediumsized half-sib families (between 20 and 65 daughters per sire) produced more accurate QTL mapping results than a small number of large families ( 5 sires with 200 progeny each) or a large number of small half-sib families (100 sires with 10 progeny each).

In sheep, linkage-based genome scans used to identify QTL underlying milk production traits have been performed using microsatellite data in various commercial (Barillet et al., 2006; Gutiérrez-Gil et al., 2009) and experimental populations (Raadsma et al., 2009; Mateescu and Thonney, 2010). The QTL detected by these classical linkage-mapping experiments are generally population specific and exhibit low significance levels and large confidence intervals (Carta et al., 2009). Based on the availability of the OvineSNP50 BeadChip (Illumina Inc., San Diego, CA), our group recently published a GWA study for milk production traits in a commercial Spanish Churra sheep population (García-Gámez et al., 2012a). In the GWA study, in addition to 14 chromosome-wise significant associations, we identified 2 overlapping experiment-wise significant associations on sheep chromosome (OAR) 3, which were coincident with a previously identified QTL for milk protein percentage in Churra sheep (Gutiérrez-Gil et al., 2009). For this QTL, the increased marker density in the GWA study allowed for the identification of the first putative causative mutation (quantitative trait nucleotide, QTN) for milk traits in dairy sheep (García-Gámez et al., 2012a). However, the analysis of the SNP array panel data set in Churra sheep demonstrated that the
LD magnitude is much shorter than observed in cattle and several other sheep breeds (García-Gámez et al., 2012c), which suggests that in this population, based on the number of markers provided by the OvineSNP50 BeadChip, the exclusive use of the LD-based analysis may demonstrate several limitations for the detection of causal mutations.

Based on this observation, the same data set used by García-Gámez et al. (2012a) was analyzed in this study to exploit the half-sib family structure of this commercial population for the identification of QTL. The results of the SNP-based whole genome scan subjected to 2 different analysis methods (the linkage analysis and LDLA approaches) were analyzed to identify the QTL influencing milk production traits. The results were compared with the GWA study reported previously (García-Gámez et al., 2012a), allowing the comparison of the 3 analysis methods and the assessment of the information that linkage-based analyses can produce in this type of commercial populations versus the use of single population-based information.

\section{MATERIALS AND METHODS}

\section{Resource Population, Phenotypes, and Marker Map}

The Spanish Churra commercial population analyzed in this study has been described in García-Gámez et al. (2012a). In total, 1,696 animals distributed in 16 halfsib families, including 1,680 ewes and the corresponding 16 rams, were commercially genotyped using the OvineSNP50 BeadChip at AROS Applied Biotechnology AS (Aarhus, Denmark) and Laboratoire d'Analyses Génétiques pour les Espèces Animales (LABOGENA; Jouy-en-Josas, France). After a quality control analysis per animal (call rate $>90 \%$ ) and per SNP (call rate $>95 \%$; minor allele frequency $>0.05$; correspondence with Hardy-Weinberg equilibrium: $P>0.00001), 1,681$ animals, including the 16 rams and 43,784 autosomal SNP, were retained for the analysis.

The phenotypes included in the analysis were the yield deviations (YD) corresponding to the milk production traits routinely collected by the National Association of Churra Breeders as follows: milk yield (MY), protein percentage $(\mathbf{P P})$, fat percentage $(\mathbf{F P})$, protein yield (PY), and fat yield (FY). The YD estimates were calculated following a multivariate animal repeatability model in which the raw phenotypes were corrected for the environmental effects of herd test day, birth order, age of the ewe at parturition (as a covariate nested within birth order), number of born lambs, number of weeks of milk production of the ewe, and the ewe's permanent environmental effect. 
The marker order and positions were based on the Ovine Assembly v2.0 (http://www.livestockgenomics. csiro.au/cgi-bin/gbrowse/oarv2.0/). This information has been previously used to evaluate the correspondence between physical and genetic maps in this Spanish Churra sheep population (García-Gámez et al., 2012c). Hence, the physical map used for the QTL detection in this study was the same as that reported in García-Gámez et al. (2012c). The equivalence $1 \mathrm{cM} \approx 1$ $\mathrm{Mb}$ was used to convert physical into genetic distances.

\section{QTL Analysis}

The QTLMap software (Filangi et al., 2010), which performs interval mapping based on maximum likelihood calculations, was used to perform the genome scans using the linkage analysis and LDLA methods. For both analyses, the QTL search was performed every $0.1 \mathrm{cM}$ using the software analysis options corresponding to linkage analysis and LDLA for a single trait (options -calcul 4 and-calcul 28, respectively). In the linkage analysis, the QTL and polygenic effects of the sires were estimated. The significance thresholds were estimated using 1,000 permutations at 5 -cM steps for the linkage analysis. To estimate the confidence interval in the linkage analysis approach, we converted the likelihood ratio test (LRT) values into logarithm odds ratio (LOD) score values and used the 1-LOD drop-off method as described in García-Gámez et al. (2012b). For the chromosome-wise significant QTL (chromosome-trait combination) identified in the linkage analysis, within-family analyses and permutationtests were performed individually for each family. The families demonstrating a 5\% chromosome-wise significant QTL were considered segregating for that specific genetic effect. The magnitude of the QTL effect in trait units was obtained, for each family, from the QTLMap output. This effect was also estimated in phenotypic standard units (SD) of the YD for the trait.

In total, 4 SNP haplotypes were constructed in the LDLA, with the QTL being placed in the central point of the haplotype. In this analysis, in addition to the effects considered in the linkage analysis model, an effect was assigned to each of the constructed haplotypes. For the LDLA, 1,000 simulations at 5-cM steps were used to set the chromosome- and genome-wise thresholds. For both linkage analysis and LDLA, we considered a QTL to be genome-wise significant if it demonstrated a chromosome-wise $P$-value $<0.0019$, as this value corresponded to a genome-wise $P$-value of 0.05 (chromosome-wise $P$-value $\times 26$ chromosomes).

We later compared the results obtained from the linkage analysis and LDLA methodological approaches presented here, and also with the results reported in the GWA study of the same data set (García-Gámez et al., 2012a). For those chromosomes showing genomewise significant associations according to at least 2 of the compared methodologies, the linkage analysis, LDLA, or both approaches were repeated by including the genotype of the most significantly associated SNP, according to the GWA analysis, in the QTL model as a fixed effect. These analyses were performed with the aim of assessing the possible direct relationship of the tested SNP with the QTL effect identified.

\section{RESULTS}

The descriptive data for the genotypes obtained using the OvineSNP50 BeadChip in the Churra sheep population analyzed, including the number of SNP per chromosome, the distance between them, the average minor allele frequency, and the heterozygosity and LD calculations, have been reported previously by GarcíaGámez et al. (2012c). A summary of the genome- and chromosome-wise significant QTL identified using the genome scans reported in this study is shown in Table 1 and Table 2 for the linkage analysis and LDLA, respectively. The results from the within-family analysis, including the estimation of the QTL effect at the position of the maximum likelihood observed for each family, are given in Table 1.

\section{Linkage Analysis}

The linkage analysis procedure identified 3 genomewise significant QTL on OAR2 for MY, PY, and FY (Table 1; Figure 1). The location of the maximum LRT value was coincident for the MY and PY QTL (at 55.1 $\mathrm{cM}$ ) and was only $1.3 \mathrm{cM}$ away from the FY QTL (at $53.8 \mathrm{cM}$ ). The confidence interval calculated following the LOD drop-off method covered $0.8 \mathrm{cM}$ of the chromosome for MY and PY and a region of $5.9 \mathrm{cM}$ for FY. Considering the results of the within-family analysis, only 1 of the families studied was segregating for the 3 QTL effects (family 1444; $5 \%$ chromosome-wise). Another segregating family was found for 2 of the OAR2 QTL (MY and PY; family 2387) although, in this case, the maximum LRT values were found in completely different regions of the chromosome (243 and 35.6 $\mathrm{cM}$, respectively). Taking into account the segregating families identified for each of these 3 significant linkage associations, the average size of the QTL effect in standard deviation units was $0.35,0.37$, and 0.465 for MY, PY, and FY, respectively.

Significant results at the $1 \%$ chromosome-wise significance level were identified in 3 genomic regions: at the proximal end of OAR10 for FP $(10 \mathrm{cM})$, at the proximal end of OAR16 for MY (12.2 cM), and within 
Table 1. Significant results for the linkage analysis performed for the milk production traits in the Spanish Churra sheep population studied in the present work

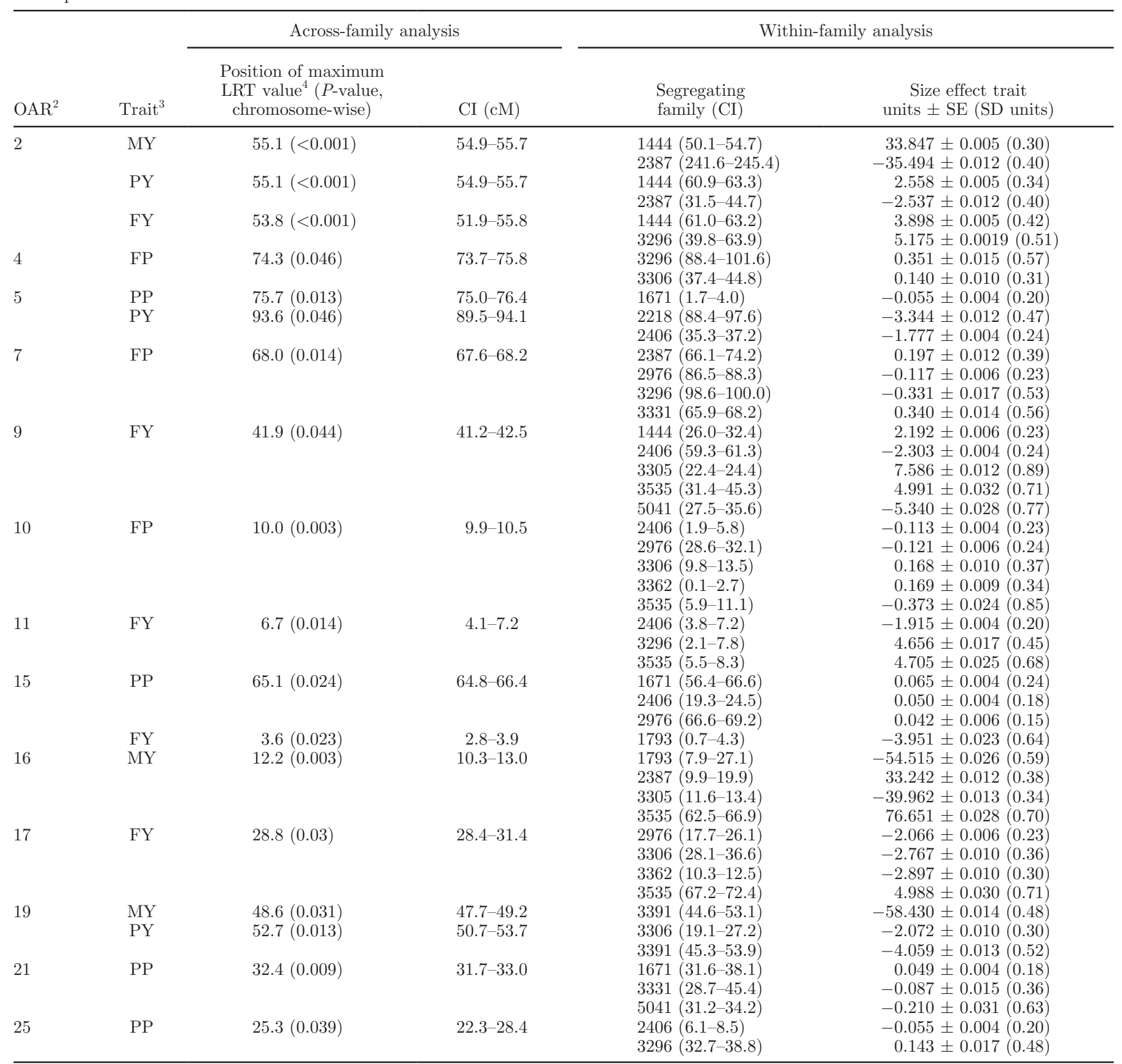

${ }^{1}$ The results for the across- and within-families analyses are reported here.

${ }^{2} \mathrm{OAR}=$ sheep chromosome.

${ }^{3} \mathrm{MY}=$ milk yield; $\mathrm{PY}=$ protein yield $; \mathrm{FY}=$ fat yield; $\mathrm{FP}=$ fat percentage; $\mathrm{PP}=$ protein percentage.

${ }^{4} \mathrm{LRT}=$ likelihood ratio test. Position is given in centimorgans.

the first third of OAR21 for PP $(32.4 \mathrm{cM}$; see Table 1 and Figure 1). For these QTL, 5, 4, and 3 segregating families were identified, respectively, with the size of QTL effect estimated in the within-family analyses ranging from 0.23 (for the FP OAR10 QTL; family
2406 ) to 0.85 (for the same FP OAR10 QTL; family 3535) standard deviation units (Table 1).

Finally, 12 additional chromosomal regions demonstrated a 5\% chromosome-wise significant linkage association with several of the traits studied. The char- 
Table 2. Genome-wise significant results of the combined linkage disequilibrium and linkage analysis (LDLA) performed for the milk production traits in the Spanish Churra sheep population analyzed in the present study

\begin{tabular}{|c|c|c|c|c|}
\hline $\mathrm{OAR}^{1}$ & Trait $^{2}$ & $\begin{array}{l}\text { Position of maximum } \\
\text { LRT value }(\mathrm{cM})\end{array}$ & $\begin{array}{l}\text { Significant } \\
\text { region }^{4}(\mathrm{cM})\end{array}$ & $\begin{array}{c}P \text {-value } \\
\text { (chromosome-wise) }\end{array}$ \\
\hline 1 & PP & 51.1 & $51.1-51.2$ & $<0.001$ \\
\hline \multirow[t]{4}{*}{2} & FY & 50.3 & $47.8-65.4$ & $<0.001$ \\
\hline & MY & 57.1 & $52.3-65.4$ & $<0.001$ \\
\hline & $\mathrm{PP}$ & 32.2 & $32.2-208.2$ & $<0.001$ \\
\hline & PY & 56.6 & $46.6-70.1$ & $<0.001$ \\
\hline \multirow[t]{3}{*}{3} & FP & 134.5 & $134.5-194.0$ & $<0.001$ \\
\hline & MY & 97 & & $<0.001$ \\
\hline & $\mathrm{PP}$ & 137.3 & $129.3-140.6$ & $<0.001$ \\
\hline 5 & $\mathrm{PP}$ & 75 & & $<0.001$ \\
\hline \multirow[t]{3}{*}{7} & FP & 67.3 & $60.8-86.5$ & $<0.001$ \\
\hline & FY & 83.4 & & $<0.001$ \\
\hline & MY & 40.6 & & $<0.001$ \\
\hline 9 & FY & 35.4 & $35.4-50.3$ & $<0.001$ \\
\hline 10 & $\mathrm{FP}$ & 8.1 & $2.8-10.0$ & $<0.001$ \\
\hline \multirow[t]{3}{*}{13} & FY & 23.5 & & $<0.001$ \\
\hline & MY & 13 & & $<0.001$ \\
\hline & $\mathrm{PP}$ & 37.4 & $25.0-37.4$ & $<0.001$ \\
\hline 15 & $\mathrm{PP}$ & 56.7 & & $<0.019$ \\
\hline \multirow[t]{3}{*}{16} & MY & 11.4 & $10.2-14.0$ & $<0.001$ \\
\hline & PP & 42.5 & & $<0.001$ \\
\hline & PY & 12.4 & & $<0.001$ \\
\hline \multirow[t]{2}{*}{17} & FY & 15.9 & $15.7-30.9$ & $<0.001$ \\
\hline & MY & 15.9 & $15.9-42.5$ & $<0.001$ \\
\hline 18 & PP & 33.4 & & $<0.019$ \\
\hline \multirow[t]{2}{*}{19} & $\mathrm{PP}$ & 28.2 & $27.4-28.2$ & $<0.019$ \\
\hline & PY & 51.8 & & $<0.001$ \\
\hline \multirow[t]{2}{*}{20} & FP & 42.5 & & $<0.001$ \\
\hline & $\mathrm{PP}$ & 0.6 & & $<0.001$ \\
\hline \multirow[t]{3}{*}{22} & FY & 47.6 & & $<0.019$ \\
\hline & MY & 6.6 & & $<0.001$ \\
\hline & PY & 7 & & $<0.001$ \\
\hline 23 & $\mathrm{FP}$ & 5.7 & $5.6-5.7$ & $<0.001$ \\
\hline 25 & $\mathrm{FP}$ & 38.8 & & $<0.019$ \\
\hline 26 & $\mathrm{PP}$ & 8.5 & $8.5-8.6$ & $<0.001$ \\
\hline
\end{tabular}

${ }^{1} \mathrm{OAR}=$ sheep chromosome.

${ }^{2} \mathrm{PP}=$ protein percentage $\mathrm{FY}=$ fat yield $\mathrm{MY}=$ milk yield $\mathrm{PY}=$ protein yield $\mathrm{FP}=$ fat percentage.

${ }^{3} \mathrm{LRT}=$ likelihood ratio test.

${ }^{4}$ Significant region: a map interval is provided for those cases where more than 1 map location exceeded the $5 \%$ genome-wise significance threshold for the considered trait and chromosome combination.

acterization of these QTL regions, including the acrossand within-family linkage analysis results, is shown in Table 1. Just to note that although in some cases the position of the maximum LRT values observed in the within-family analyses (not shown) and the corresponding confidence interval (Table 1) showed important discrepancies with the positions suggested by the across-family analyses, this is an intrinsic limitation of linkage analysis based on the analysis of commercial populations (Georges, 1998). Hence, the comparison of results discussed in this paper is focused on the results observed in the across-family analysis.

\section{$L D L A$}

In total, 34 genome-wise significant QTL regions distributed across 18 of the analyzed chromosomes were detected using the LDLA approach implemented in the QTLMap software (Table 2; Figure 2), whereas many other chromosome-wise significant associations were also identified (results not shown). For simplicity, we will focus on describing the genome-wise significant regions. For some of the regions, more than 1 haplotype was detected as significantly associated with the trait, and the chromosomal region including those significant haplotypes is indicated in Table 2 as "significant region".

The most significant QTL identified by the LDLA procedure was found on OAR3 for PP (Figure 2b). The maximum LRT value for this QTL was found at 137.3 $\mathrm{cM}$, whereas the region involving significant LRT values above the $5 \%$ genome-wise significance threshold was between 129.3 and $140.6 \mathrm{cM}$. In the same chromosomal region, a QTL associated with FP was identified. For 

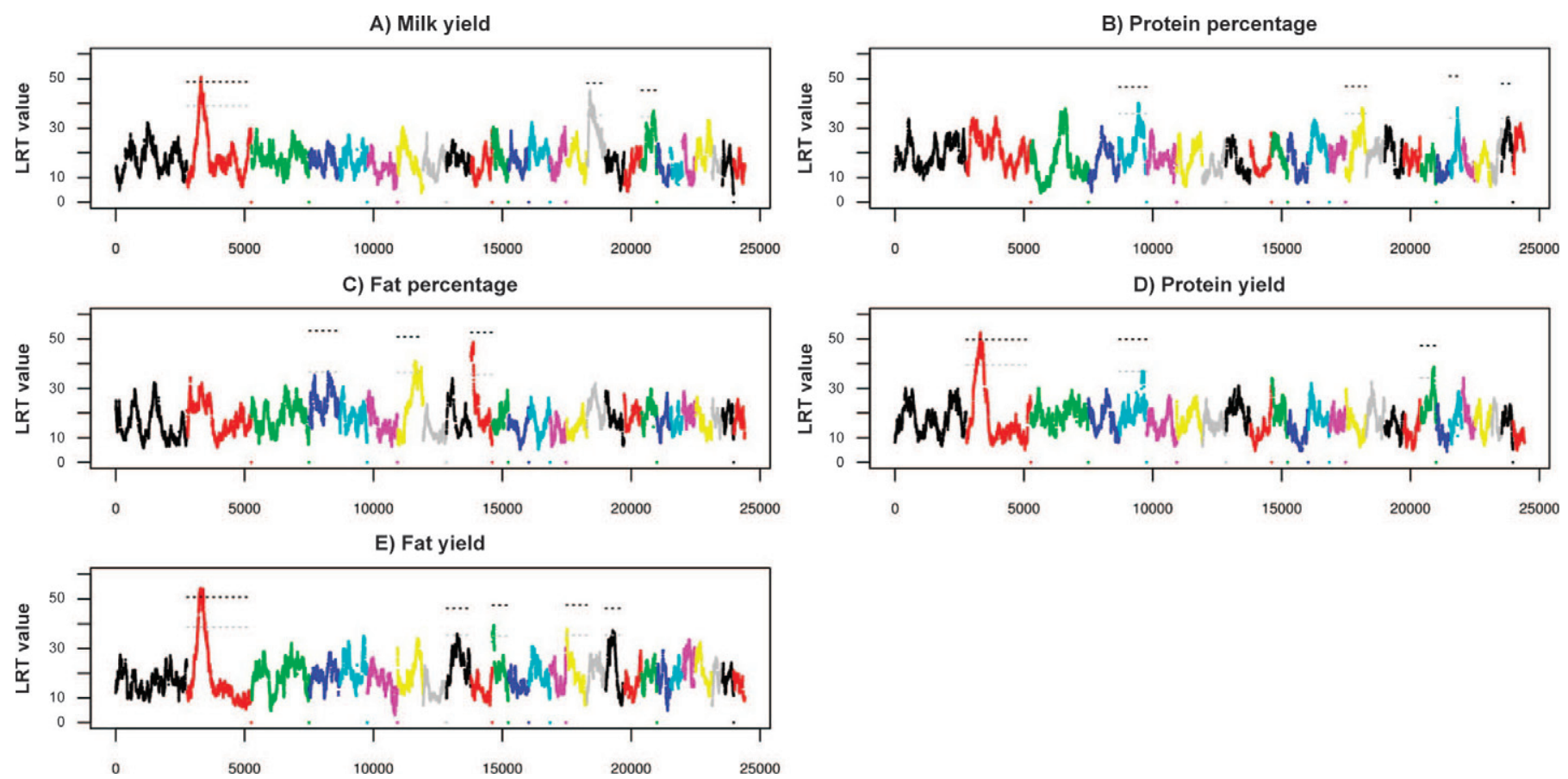

Figure 1. Linkage analysis scan of the sheep genome for the 5 milk production traits analyzed in this study in a commercial population of Spanish Churra sheep. The $\mathrm{x}$ - and $\mathrm{y}$-axes represent the scan steps of the analysis (at $0.1 \mathrm{cM}$ ) and the likelihood ratio test (LRT value), respectively. Color version available in the online PDF.

the FP trait, the maximum LRT value was located at $134.5 \mathrm{cM}$, whereas other significant LRT values for this trait were found between 137.2 and $137.5 \mathrm{cM}$, overlapping with the maximum LRT value identified for PP.

Whereas these OAR3 QTL were not identified by the linkage analysis described above, several QTL were identified in common through the linkage analysis and LDLA methodologies. For example, the region on OAR2 identified at the genome-wise significance level by the linkage analysis scan was also found significantly associated, at the $5 \%$ genome-wise significance level, with MY, PY, and FY, following the LDLA approach (Figure 2). In this case, the maximum LRT values detected for the 3 traits were close to the QTL peaks detected using the linkage analysis. Additionally, the QTL segregating for MY on OAR16 was identified using the LDLA procedure, with the maximum located at $11.4 \mathrm{cM}$, which was within the confidence interval estimated based on the linkage analysis procedure. The LDLA scan also identified a QTL associated with FY in the central region of OAR9 $(50.3 \mathrm{cM})$, which was close to the chromosome-wise significant QTL detected for the same trait using the linkage analysis. The region on OAR10 influencing FP was also identified using the linkage analysis and LDLA methods. Although the maximum LRT value (at position $8.1 \mathrm{cM}$ ) identified by the LDLA was not within the confidence interval estimated using the linkage analysis method, it was 1.8 $\mathrm{cM}$ proximal to the linkage analysis value. In addition, the maximum LRT value for a QTL influencing FP on OAR7 was located at $67.3 \mathrm{cM}$ and exhibited genomewise significant results in the surrounding region (from 60.8 to $86.5 \mathrm{cM})$.

Of the results obtained using the LDLA scan, additional chromosomes demonstrated genome-wise significant associations with the traits studied that had not been identified by linkage analysis (see Table 2 for details). Trying to provide a global overview of the comparison of the results obtained through the 2 methodologies used in this work, a summary table is provided in Figure 3. We also provide in this figure a comparison with the significant results reported in the GWA study previously reported for the same data set (García-Gámez et al., 2012a).

\section{QTL Detection Including SNP Genotypes in the Model}

The summary presented in Figure 3 shows that only 2 QTL were detected at the genome-wise level by at least 2 of the 3 methods: the QTL identified for PP and FP on OAR3, and the OAR2 QTL influencing the 3 milk yield traits MY, PY, and FY. For these 2 chromosomes, further analyses were performed by fix- 
A) Milk yield

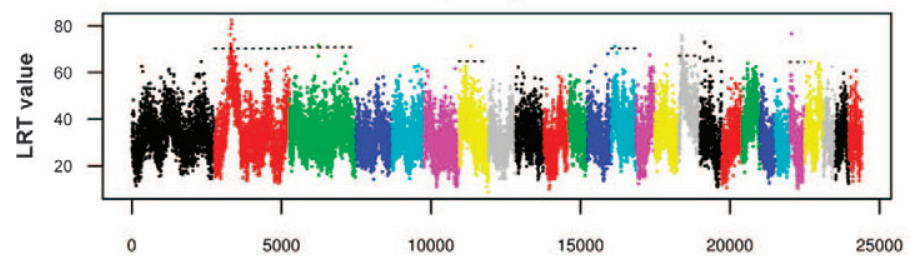

C) Fat percentage
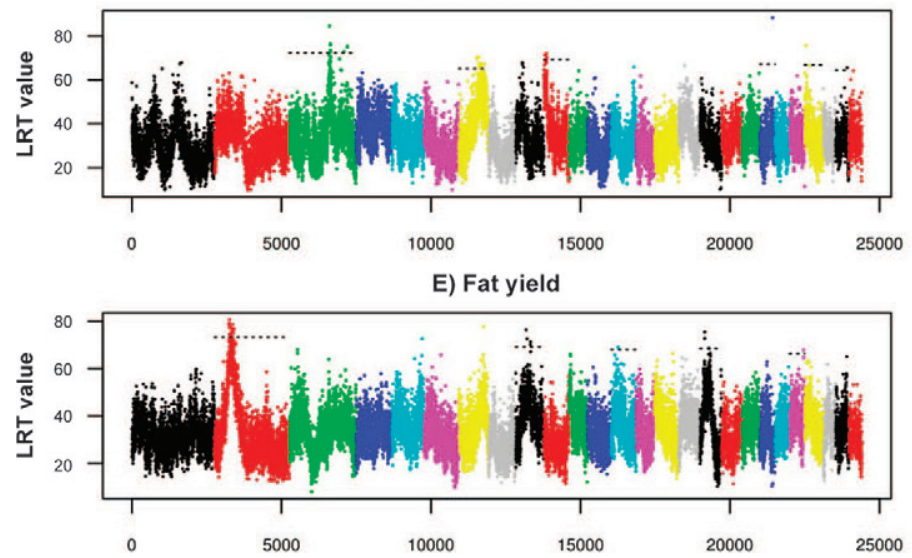

B) Protein percentage

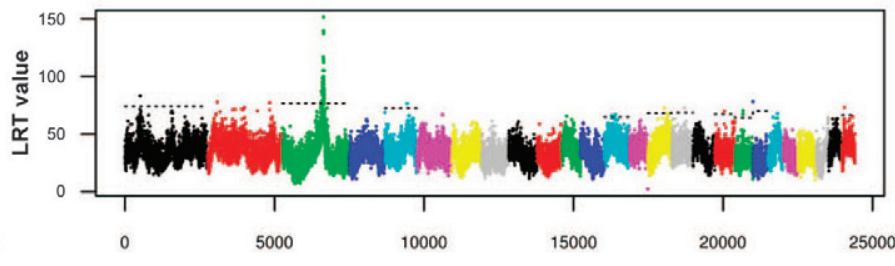

D) Protein yield

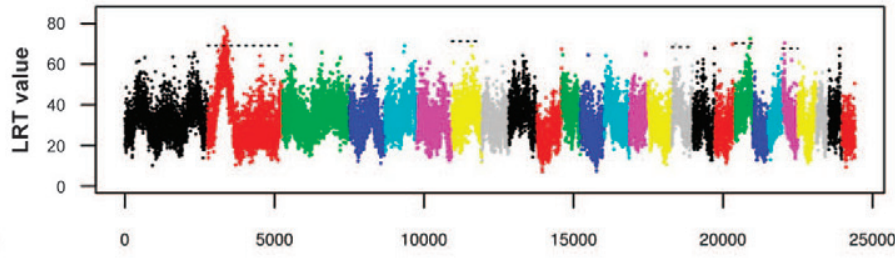

Figure 2. Statistical profile of the likelihood ratio test (LRT) obtained using the combined linkage disequilibrium and linkage analysis (LDLA) approach for the 5 milk production traits analyzed in this study in a commercial population of Spanish Churra sheep. The x- and y-axes represent the scan steps of the analysis $($ at $0.1 \mathrm{cM})$ and the LRT value, respectively. Color version available in the online PDF.

ing, as a fixed effect, in the corresponding QTL model (linkage analysis, LDLA, or both models) the most significant SNP identified in the GWA analysis reported by García-Gámez et al. (2012a).

Regarding the OAR3 QTL, the genotype of the putative QTN proposed for the OAR3 PP QTL in García-Gámez et al. (2012a), a mutation in the $\alpha$-LA $(\boldsymbol{L} \boldsymbol{A} \boldsymbol{L} \boldsymbol{B} \boldsymbol{A})$ gene $\left(L A L B A \_g .242 \mathrm{~T}>\mathrm{C}\right)$, was added as a fixed effect for the traits showing significant results in the initial LDLA scan, PP, and FP. Notably, this SNP was not included in the SNP array panel but was identified at a later stage when the $L A L B A$ gene was analyzed exhaustively as a strong candidate gene for the PP QTL effect (García-Gámez et al. (2012a). The results obtained in the repeated LDLA analysis on OAR3 differed from the results obtained using the model without the fixed effects assigned to the SNP genotypes because the 2 significant QTL for PP and FP on OAR3 were cancelled (Supplementary Figure S1; http://dx.doi.org/10.3168/jds.2013-6824).

Following the same approach, for the OAR2 QTL, the genotypes fixed in these additional analyses were the SNP demonstrating the highest association on OAR2 for each of the yield-related traits in accordance with the GWA analysis reported by García-Gámez et al. (2012a). Therefore, the markers OAR2_59276935, s25113, and OAR2_67323597 were fixed in the re- peated scans of MY, PY, and FY, respectively. By repeating the linkage analysis with these SNP fixed, the significance of the MY QTL on OAR2 decreased from the genome-wise significance level to the $5 \%$ chromosome-wise level, whereas the results for the PY and FY remained similar to those obtained using the model without the fixed SNP (Supplementary Figure S2; http://dx.doi.org/10.3168/jds.2013-6824). Fixing the OAR2 SNP in the corresponding LDLA models also demonstrated several differences compared with the initial LDLA scan. In this case, the significance of the PY and FY OAR2 QTL decreased from the 5\% genome-wise to the $1 \%$ chromosome-wise level, whereas the QTL influencing MY remained significant at the $5 \%$ genome-wise significance level, although in this case, the significant association was identified only for a single map position (57.1 cM; Supplementary Figure 3; http://dx.doi.org/10.3168/jds.2013-6824).

\section{DISCUSSION}

In recent years, classical QTL linkage experiments based on microsatellite markers have been reported for milk production traits in different sheep populations (reviewed in Arranz and Gutiérrez-Gil, 2012). The development of a cost-efficient, high-throughput SNP genotyping platform for sheep will require the paral- 


\begin{tabular}{|c|c|c|c|}
\hline Chromosome & Linkage analysis & LDLA & GWA \\
\hline OAR01 & & PP & $\mathrm{MY}^{\mathrm{a}}$ \\
\hline \begin{tabular}{|l|} 
OAR02 \\
\end{tabular} & MY.PY.FY & $\mathrm{MY}, \mathrm{PY}, \mathrm{FY}, \mathrm{PP}^{\mathrm{a}}$ & MY, PY, FY, MY ${ }^{b}, P^{c}$ \\
\hline OAR03 & & $M Y^{a}, P P, F P$ & $\begin{array}{ll}\mathrm{PP}, \mathrm{FP}, & \mathrm{FY}^{\mathrm{b}} \\
\end{array}$ \\
\hline OAR04 & FP & & PY \\
\hline OAR05 & PP. PY ${ }^{\mathrm{a}}$ & $\mathrm{PP}$ & \\
\hline \begin{tabular}{|l|} 
OAR06 \\
\end{tabular} & & & PP \\
\hline \begin{tabular}{|l|} 
OAR07 \\
\end{tabular} & $\mathrm{FP}^{\mathrm{a}}$ & MY. FP ${ }^{a} . F Y^{b}$ & $\mathrm{PY}^{\mathrm{b}}$ \\
\hline \multicolumn{4}{|l|}{ OAR08 } \\
\hline OAR09 & FY & FY & \\
\hline OAR10 & FP & FP & \\
\hline OAR11 & FY & & \\
\hline OAR12 & & & MY \\
\hline OAR13 & & $M Y^{a}, P P . F Y$ & \\
\hline OAR14 & & & PP, FY, PP $, F^{\mathrm{a}}, \mathrm{P}^{\mathrm{b}}$ \\
\hline OAR15 & FY. PP ${ }^{\mathrm{a}}$ & $\mathrm{PP}^{\mathrm{a}}$ & $\mathrm{PP}^{\mathrm{a}}$ \\
\hline OAR16 & MY & MY, PY, PP & $\mathrm{MY}^{\mathrm{b}}, \mathrm{PP}^{\mathrm{a}}$ \\
\hline OAR17 & FY & MY, FY & PP. FP ${ }^{a}$ \\
\hline OAR18 & & $\mathrm{PP}$ & \\
\hline OAR19 & MY.PY & PP'. PY & \\
\hline \begin{tabular}{|l|} 
OAR20 \\
\end{tabular} & & PP. FP & $\mathrm{PP}^{\mathrm{b}}, \mathrm{FP}^{\mathrm{b}}, \mathrm{FY}^{\mathrm{b}}$ \\
\hline OAR21 & PP & & \\
\hline OAR22 & & MY. PY. FY & \\
\hline OAR23 & & FP & $\mathrm{PP}^{\mathrm{a}} \cdot \mathrm{PY}^{\mathrm{b}}$ \\
\hline \multicolumn{4}{|l|}{ OAR24 } \\
\hline OAR25 & PP & FP & FY \\
\hline OAR26 & & PP & \\
\hline
\end{tabular}

\section{Significance level}

\section{$5 \%$ genome-wise}

$1 \%$ chromosome-wise

$5 \%$ chromosome-wise

Figure 3. Summary table of the significant results detected using linkage analysis, a combination of linkage disequilibrium and linkage analysis (LDLA), and a genome-wide association (GWA) analysis for milk production traits in the commercial population of Spanish Churra sheep analyzed in this study. The results from the linkage analysis and LDLA methodology analyses are reported here, whereas the results from the GWA study have been taken from García-Gámez et al. (2012a). A color code is used to show the statistical significance achieved for the genetic effects identified: $5 \%$ genome-wise (red; very dark gray), $1 \%$ chromosome-wise (dark gray), and $5 \%$ chromosome-wise (light gray). The traits analyzed were milk yield $(\mathrm{MY})$, protein percentage $(\mathrm{PP})$, fat percentage $(\mathrm{FP})$, protein yield $(\mathrm{PY})$, and fat yield $(\mathrm{FY})$. OAR = sheep chromosome. ${ }^{\text {a-c }}$ Different superscript letters indicate different map position of the corresponding QTL, with regard to the other QTL located in the same chromosome. Color version available in the online PDF. 
lel evolution of QTL mapping strategies. Whereas our research group has recently reported the first ovine GWA study performed for milk production traits (García-Gámez et al., 2012a) using genotypes from the OvineSNP50 BeadChip, in this study, we summarized the results of the first linkage analysis and LDLA genome scans for milk production traits based on these data.

The 2 analysis methodologies used in this study, linkage analysis and LDLA, yielded different results, although significantly associated regions common to both procedures were identified. Linkage analysis, which exploits family information, has been the traditional method used for QTL mapping. The investigation of LD through GWA analyses, which takes into account population information, has begun to replace linkage analysis because marker maps are now dense enough to exploit LD across the genome. Finally, the LDLA procedure, implemented using the QTLMap software, based on Legarra and Fernando (2009), uses all available information (family and population data) in an approach that is computationally simpler than other LDLA proposals. Moreover, this LDLA procedure has performed satisfactorily compared with other LDLA methodologies (Legarra and Fernando, 2009). An issue associated with performing the LDLA is the haplotype length chosen. In a simulation study, haplotypes of 4 or 6 markers demonstrated higher mapping accuracy compared with longer or shorter haplotype lengths (Grapes et al., 2006). The QTLMap software uses a fixed length of 4-SNP haplotypes to perform the LDLA.

The detailed comparison of the data reported in the current study, based on a half-sib population, demonstrated that the linkage analysis method identified only a genome-wise significant QTL region, whereas for the same level of significance, the LDLA method identified 34 regions. Although this suggested that the LDLA method represents an important improvement in the QTL detection compared with traditional linkage analysis, as suggested by Farnir et al. (2002) and Meuwissen et al. (2001, 2002), we cannot ignore that the differences in the estimation of the significance thresholds through permutation or simulation could explain these findings. To assess this hypothesis, we compared the chromosome-wise significant QTL detected using the linkage analysis method with the genome-wise significant QTL detected using the LDLA method and identified 11 QTL detected by the 2 methods. In contrast, 33 QTL were detected using either the linkage analysis (7) or LDLA (26) methods. The regions identified exclusively using the linkage analysis method are likely to correspond to low-frequency QTL segregating within several of the analyzed families. However, the 26 regions identified using the LDLA method that were not identified in the linkage analysis method indicate that the LDLA method may provide an important improvement in the power and efficiency of QTL detection in half-sib families, as suggested previously (Farnir et al., 2002; Meuwissen et al., 2002). Therefore, the use of the population-based information in combination with the family-based information appears to be a useful method for the analysis of resource populations that provide the appropriate structure, such as the population analyzed in the current study.

The identification of 3 overlapping QTL effects identified at approximately $55 \mathrm{cM}$ on OAR2 for MY, PY, and FY, with at least 1 common segregating family identified for the 3 effects (family 1444), suggested the presence of a single pleiotropic QTL in this region influencing the 3 yield-related traits studied. For family 1444, the coincident sign of the effects of the 3 QTL suggests that the pleiotropic QTL on OAR2 influences in the same direction the 3 yield-related traits analyzed here and, therefore, is not responsible for a dilutionconcentration effect. This pleiotropic QTL on OAR2 was also detected at the genome-wise significance level using LDLA and at the chromosome-wise level in the GWA analysis performed on the same Spanish Churra population (García-Gámez et al., 2012a). The fact that this pleiotropic QTL was detected using the linkage analysis, LD, and LDLA methods indicates that it was segregating at both the across-population and within-family levels. As indicated by García-Gámez et al. (2012a), this region was associated with various milk traits in the microsatellite-based genome scan performed in Spanish Churra sheep (Gutiérrez-Gil et al., 2009). Other associations between this region and the milk production traits have not been reported in other sheep populations. In addition to the positional candidate genes indicated by García-Gámez et al. (2012a) for this QTL, among which insulin-like growth factor binding protein 1 (IGFBPL1) was a strong candidate, we suggest here another promising functional candidate gene, the gene encoding the guanine nucleotide-binding protein q polypeptide (GNAQ protein). Haplotypes including this gene in humans and mice play a role in maternal nurturing ability either through their potential effect on insulin signaling within the mammary gland or through their influence on maternal behavior (Hadsell et al., 2012).

The fact that for these QTL on OAR2, the fixation of the SNP selected based on the GWA analysis (GarcíaGámez et al., 2012a) resulted for both methods, linkage analysis and LDLA, in a substantial decrease of the QTL significance (for some of these traits) suggests that the fixed SNP are in linkage disequilibrium with the causal mutation but not directly responsible of the QTL effects. A higher marker density and the investi- 
gation of additional animals will be needed to decipher the true nature of the OAR2 QTL effects reported in Spanish Churra sheep.

The most significant associations identified by the LDLA, the QTL for PP and FP on OAR3, were not detected by the linkage analysis scan. These QTL were described previously using microsatellite data (Gutiérrez-Gil et al., 2009) and were replicated and refined (García-Gámez et al., 2012b), and a QTN responsible for the detected effects was recently proposed (GarcíaGámez et al., 2012a). As discussed in García-Gámez et al. (2012a), these coincident QTL also suggested the presence of a pleiotropic QTL influencing 2 correlated milk composition traits. Protein and fat percentages display a phenotypic correlation of 0.62 in Churra sheep (Othmane et al., 2002).

When the LDLA genome scan was repeated by adding the genotypes of the putative QTN for the PP QTL (the LALBA_g.242T >C mutation proposed by GarcíaGámez et al., 2012a) as fixed factors in the analysis model, the OAR3 QTL for PP and FP were cancelled (Supplementary Figure S1; http://dx.doi.org/10.3168/ jds.2013-6824). This is consistent with the observations reported in the GWA analysis description (GarcíaGámez et al., 2012a) and supports that the QTN is directly responsible for the effects detected by the LDLA analysis.

Among the other QTL reported in this study, and independently of the significance level, 8 regions were significantly detected by the 2 approaches (linkage analysis and LDLA): OAR5 (PP), OAR7 (FP), OAR9 (FY), OAR10 (FP), OAR15 (PP), OAR16 (MY), OAR17 (FY), and OAR19 (PY; see Supplementary Figure S1; http://dx.doi.org/10.3168/jds.2013-6824). When looking for correspondence of these regions with other QTL previously reported in dairy sheep, some interesting coincidences have been found. The QTL identified for PP on OAR5 is close to a QTL influencing the same trait reported in Lacaune and Manech breeds (Barillet et al., 2006), whereas the OAR7 QTL for FP maps close to 2 QTL described previously for FP and PP in a Sarda $x$ Lacaune experimental design (Barillet et al., 2006) and for PP in an Awassi $\times$ Merino population (Raadsma et al., 2009). The diacylglycerol O-acyl-transferase 1 gene (DGAT1), which harbors a QTN for milk fat traits in dairy cattle, maps to the proximal end of OAR9 [at $13.52 \mathrm{Mb}$, according to the Ovine Genome Assembly v2.0 (http://www.livestockgenomics.csiro.au/cgi-bin/ gbrowse/oarv2.0/)]. This gene has been proposed as candidate gene of other QTL identified for PP and FP in Lacaune and Manech commercial populations (Barillet et al., 2006). However, the FY QTL effects reported here map distal to this gene, which agrees with a previous association study performed in Spanish Churra that did not identify a significant association of DGAT1 with milk production traits (García-Fernández et al., 2011). For the remaining QTL located on OAR10 (FP), OAR15 (PP), OAR16 (MY), OAR17 (FY), OAR19 (PY), no correspondence with previous QTL affecting dairy traits in sheep has been found. The large number of positional candidates located within the estimated confidence interval with no clear functional candidates suggests that further efforts are needed to confirm these putative QTL and to refine the confidence interval to better define these genetic effects.

Overall, the results reported in this study represent the first genome scan based on high-throughput SNP genotyping in which linkage information, by itself or combined with LD, was exploited in dairy sheep. The results presented here suggest that in this dairy sheep resource population, which exhibits a half-sib family structure, the LDLA analysis might be more informative than the other 2 methods because it exploits both the linkage information obtained at the within-family level and the LD information obtained at the population level.

\section{CONCLUSIONS}

The 2 methodologies used in this study detected QTL previously described and new QTL affecting milk production traits. Although we could suggest that the LDLA method provided a higher QTL-detection efficiency than simple linkage analysis and LD in family-structured populations, we believe that comparing the 3 methods is the best approach for obtaining a global picture of all identifiable QTL segregating in the population at both family-based or population-based levels, producing a better understanding of the complex genetic architecture underlying milk production traits.

\section{ACKNOWLEDGMENTS}

This work was supported by the Spanish Ministry of Science (Madrid, Spain; Project AGL2009-07000) and by the European Commission (Brussels, Belgium) by the 3SR Project (Sustainable Solutions for Small Ruminants; http://www.3srbreeding.eu). The support and availability of the computing facilities of the Foundation of Supercomputing Center of Castile and León (FCSCL; http://www.fcsc.es) are greatly acknowledged.

\section{REFERENCES}

Andersson, L. 2009. Genome-wide association analysis in domestic animals: A powerful approach for genetic dissection of trait loci. Genetica 136:341-349.

Arranz, J. J., and B. Gutiérrez-Gil. 2012. Detection of QTL underlying milk traits in sheep: An update. Chapter 5 in Milk Pro- 
duction-Advanced Genetic Traits, Cellular Mechanism, Animal Management and Health. N. Chaiyabutr, ed. InTech, New York, NY. Accessed June 2013. http://www.intechopen.com/books/ milk-production-advanced-genetic-traits-cellular-mechanism-animal-management-and-health/detection-of-qtl-underlying-milktraits-in-sheep-an-update.

Barillet F., Arranz J.J., Carta A., Jacquiet P., Stear M., and Bishop S. 2006. Final consolidated report of the European Union contract of acronym "genesheepsafety". QTLK5-CT-2000-00656, p. 145.

Carta, A., S. Casu, and S. Salaris. 2009. Invited review: Current state of genetic improvement in dairy sheep. J. Dairy Sci. 92:58145833 .

Farnir, F., B. Grisart, W. Coppieters, J. Riquet, P. Berzi, N. Cambisano, L. Karim, M. Mni, S. Moisio, P. Simon, D. Wagenaar, J. Vilkki, and M. Georges. 2002. Simultaneous mining of linkage and linkage disequilibrium to fine map quantitative trait loci in outbred half-sib pedigrees: Revisiting the location of a quantitative trait locus with major effect on milk production on bovine chromosome 14. Genetics 161:275-287.

Filangi, O., C. Moreno, H. Gilbert, A. Legarra, P. Le Roy, and J. M. Elsen. 2010. QTLMap, a software for QTL detection in outbred populations. Communication 0787 in Proc. 9th World Congr. Genet. Appl. Livest. Prod., Leipzig, Germany. Gesellschaft für Tierzuchtwissenschaften, ed. Accessed June 2013. http://www. kongressband.de/wcgalp2010/assets/pdf/0787.pdf.

García-Fernández, M., B. Gutiérrez-Gil, J. P. Sánchez, J. A. Morán, E. García-Gámez, L. Álvarez, and J. J. Arranz. 2011. The role of bovine causal genes underlying dairy traits in Spanish Churra sheep. Anim. Genet. 42:415-420.

García-Gámez, E., B. Gutiérrez-Gil, G. Sahana, J. P. Sánchez, Y. Bayón, and J. J. Arranz. 2012a. GWA analysis for milk production traits in dairy sheep and genetic support for a QTN influencing milk protein percentage in the $L A L B A$ gene. PLoS ONE 7:e47782. http://dx.doi.org/10.1371/journal.pone.0047782.

García-Gámez, E., B. Gutiérrez-Gil, J. P. Sánchez, and J. J. Arranz. 2012b. Replication and refinement of a quantitative trait locus influencing milk protein percentage on ovine chromosome 3. Anim. Genet. 43:636-641.

García-Gámez, E., G. Sahana, B. Gutiérrez-Gil, and J. J. Arranz. 2012c. Linkage disequilibrium and inbreeding estimation in Spanish Churra sheep. BMC Genet. 13:43.

Georges, M. 1998. Mapping genes underlying production traits in livestock. Pages 77-101 in Animal Breeding: Technology for the 21st Century. A. J. Clark, ed. Harwood Academic Publishers, Amsterdam, the Netherlands.

Georges, M. 2007. Mapping, fine mapping, and molecular dissection of quantitative trait loci in domestic animals. Annu. Rev. Genomics Hum. Genet. 8:131-162.

Georges, M., D. Nielsen, M. Mackinnon, A. Mishra, R. Okimoto, A. T. Pasquino, L. S. Sargeant, A. Sorensen, M. R. Steele, X. Zhao, J. E. Womack, and I. Hoeschele. 1995. Mapping quantitative trait loci controlling milk production in dairy cattle by exploiting progeny testing. Genetics 139:907-920.

Grapes, L., M. Z. Firat, J. C. Dekkers, M. F. Rothschild, and R. L. Fernando. 2006. Optimal haplotype structure for linkage disequi- librium-based fine mapping of quantitative trait loci using identity by descent. Genetics 172:1955-1965.

Gutiérrez-Gil, B., M. F. El-Zarei, L. Alvarez, Y. Bayón, L. F. De la Fuente, F. San Primitivo, and J. J. Arranz. 2009. Quantitative trait loci underlying milk production traits in sheep. Anim. Genet. 40:423-434.

Hadsell, D. L., J. Wei, W. Olea, L. A. Hadsell, A. Renwick, P. C. Thomson, M. Shariflou, and P. Williamson. 2012. In silico QTL mapping of maternal nurturing ability with the mouse diversity panel. Physiol. Genomics 44:787-798.

Legarra, A., and R. L. Fernando. 2009. Linear models for joint association and linkage QTL mapping. Genet. Sel. Evol. 41:43.

Mai, M. D., G. Sahana, F. B. Christiansen, and B. Guldbrandtsen. 2010. A genome-wide association study for milk production traits in Danish Jersey cattle using a 50K single nucleotide polymorphism chip. J. Anim. Sci. 88:3522-3528.

Mateescu, R. G., and M. L. Thonney. 2010. Genetic mapping of quantitative trait loci for milk production in sheep. Anim. Genet. 41:460-466.

McRae, A. F., J. C. McEwan, K. G. Dodds, T. Wilson, A. M. Crawford, and J. Slate. 2002. Linkage disequilibrium in domestic sheep. Genetics 160:1113-1122.

Meuwissen, T. H., B. J. Hayes, and M. E. Goddard. 2001. Prediction of total genetic value using genome-wide dense marker maps. Genetics 157:1819-1829.

Meuwissen, T. H., A. Karlsen, S. Lien, I. Olsaker, and M. E. Goddard. 2002. Fine mapping of a quantitative trait locus for twinning rate using combined linkage and linkage disequilibrium mapping. Genetics 161:373-379.

Othmane, M. H., L. F. De La Fuente, J. A. Carriedo, and F. San Primitivo. 2002. Heritability and genetic correlations of test day milk yield and composition, individual laboratory cheese yield, and somatic cell count for dairy ewes. J. Dairy Sci. 85:2692-2698.

Raadsma, H. W., E. Jonas, D. McGill, M. Hobbs, M. K. Lam, and P. C. Thomson. 2009. Mapping quantitative trait loci (QTL) in sheep. II. Meta-assembly and identification of novel QTL for milk production traits in sheep. Genet. Sel. Evol. 41:45.

Roldan, D. L., H. Gilbert, J. M. Henshall, A. Legarra, and J.-M. Elsen. 2012. Fine-mapping quantitative trait loci with a medium density marker panel: Efficiency of population structures and comparison of linkage disequilibrium linkage analysis models. Genet. Res. (Camb.) 94:223-234.

Schopen, G. C., M. H. Visker, P. D. Koks, E. Mullaart, J. A. van Arendonk, and H. Bovenhuis. 2011. Whole-genome association study for milk protein composition in dairy cattle. J. Dairy Sci. 94:3148-3158

Weller, J. I., Y. Kashi, and M. Soller. 1990. Power of daughter and granddaughter designs for determining linkage between marker loci and quantitative trait loci in dairy cattle. J. Dairy Sci. $73: 2525-2537$.

Weller, J. I., and M. Ron. 2011. Invited review: Quantitative trait nucleotide determination in the era of genomic selection. J. Dairy Sci. 94:1082-1090. 